\title{
Single-Neuron RNA Modification Analysis by Mass Spectrometry: Characterizing RNA Modification Patterns and Dynamics with Single-Cell Resolution
}

\author{
Kevin D. Clark, ${ }^{1,2}$ Stanislav S. Rubakhin, ${ }^{1,2}$ Jonathan V. Sweedler ${ }^{1,2, *}$ \\ ${ }^{1}$ Beckman Institute for Advanced Science and Technology, University of Illinois at Urbana- \\ Champaign, Urbana, Illinois 61801, United States \\ ${ }^{2}$ Department of Chemistry, University of Illinois at Urbana-Champaign, Urbana, Illinois \\ 61801, United States \\ *Correspondence: jsweedle@illinois.edu
}

\section{Table of Contents}

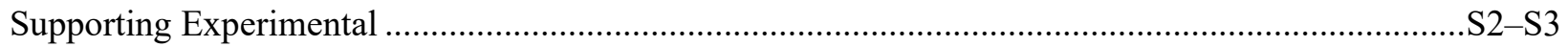

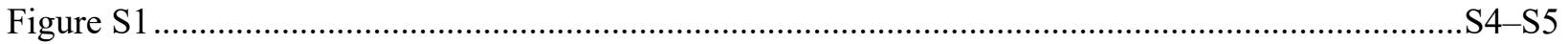

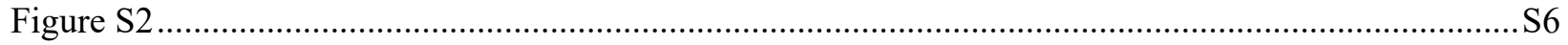

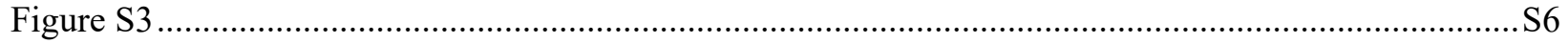

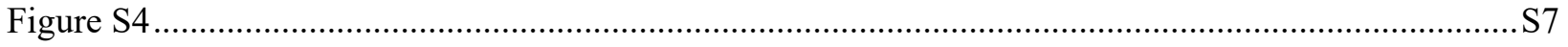

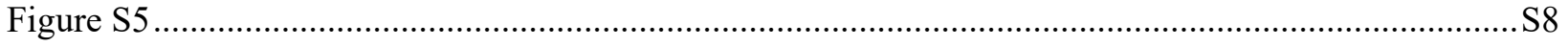

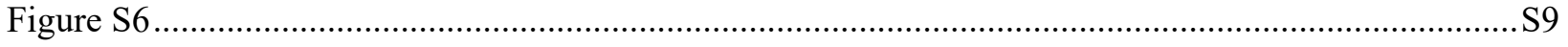

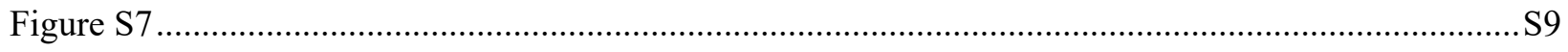

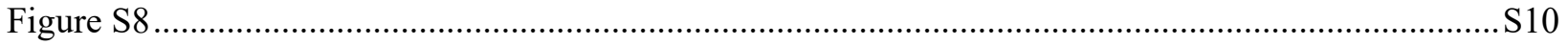

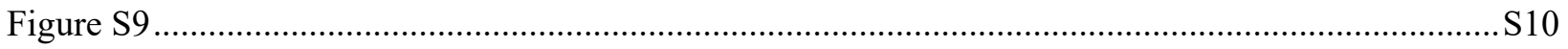

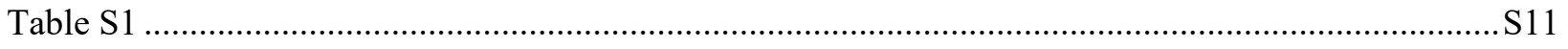

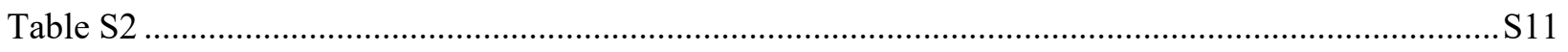

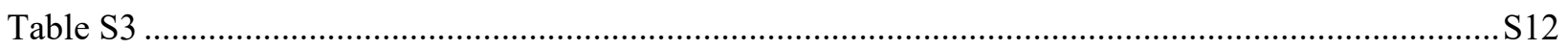

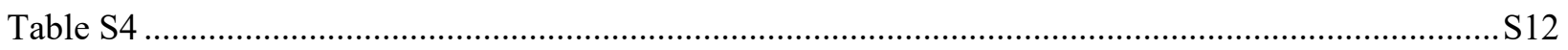

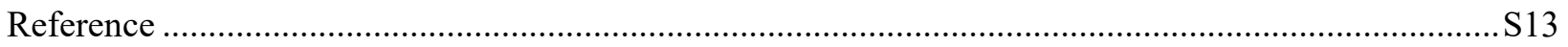




\section{Supporting Experimental}

Reagents. Magnesium chloride, sodium chloride, calcium chloride, magnesium sulfate, bovine serum albumin, penicillin $\mathrm{G}$, pentostatin, streptomycin sulfate, actinomycin D, and a nucleosides test mix were purchased from Sigma-Aldrich (St. Louis, MO). Alkaline phosphatase and phosphodiesterase I were obtained from Worthington Biochemical Corp. (Lakewood, NJ). Benzonase was purchased from EMD Millipore (Temecula, CA). Potassium chloride, gentamycin sulfate, 4-(2-hydroxyethyl)-1-piperazineethane-sulfonic acid (HEPES), chloroform, and isopropanol were purchased from Fisher Scientific (Fairlawn, NJ). L-methionine (methyl-D 3 , 98\%) was obtained from Cambridge Isotope Laboratories (Tewksbury, MA). N6-methyladenosine and 2'-O-methyladenosine were purchased from Cayman Chemical Company (Ann Arbor, MI). Trizol reagent was obtained from Life Technologies (Carlsbad, CA). Artificial seawater (ASW) was prepared with $460 \mathrm{mM} \mathrm{NaCl}, 10 \mathrm{mM} \mathrm{KCl}, 10 \mathrm{mM} \mathrm{CaCl}_{2}, 22 \mathrm{mM} \mathrm{MgCl}, 26 \mathrm{mM} \mathrm{MgSO}$,

and $10 \mathrm{mM}$ HEPES, $100 \mathrm{U} \mathrm{mL}^{-1}$ penicillin $\mathrm{G}, 100 \mu \mathrm{g} \mathrm{mL}{ }^{-1}$ streptomycin, and $100 \mu \mathrm{g} \mathrm{mL} \mathrm{m}^{-1}$ gentamicin. Milli-Q water from a Milli-Q purification system (Millipore, Bedford, MA) was used for the preparation of all solutions.

Single-Neuron RNA Extraction with Phenol-Chloroform Method. For comparison of SNRMA-MS and conventional RNA extraction for detection of RNA modifications, identified LP11 neurons were isolated and RNA extracted according to the manufacturer's instructions with slight modifications to account for the small-sample volumes. Single, isolated neurons were transferred into microcentrifuge tubes containing $10 \mu \mathrm{L}$ of Trizol reagent. Samples were vortexed for $30 \mathrm{~s}$ and then $5 \mu \mathrm{L}$ of chloroform added, followed by vortexing for another $30 \mathrm{~s}$. Samples were centrifuged at $8000 \mathrm{~g}$ and $4{ }^{\circ} \mathrm{C}$ for $5 \mathrm{~min}$ and the upper aqueous layer transferred to a clean microcentrifuge tube. Then, $50 \mu \mathrm{L}$ of isopropanol were added to the tube and the sample stored at 
$-20{ }^{\circ} \mathrm{C}$ overnight. The sample was centrifuged at $8000 \mathrm{~g}$ and $4{ }^{\circ} \mathrm{C}$ for $15 \mathrm{~min}$, supernatant decanted, and the pellet washed with $75 \%$ ethanol. The open tube was placed under a gentle stream of nitrogen for $20 \mathrm{~min}$ to evaporate residual solvent. Then, $5 \mu \mathrm{L}$ of Milli-Q water were transferred into the tube for sample resuspension.

LC-MS/MS Analysis. Gradient elution was performed with mobile phase A consisting of 5 $\mathrm{mM}$ ammonium acetate $(\mathrm{pH} 5.6)$ and mobile phase $\mathrm{B}$ as a $60 / 40$ mixture of mobile phase A/acetonitrile with the following linear gradient parameters: $0-5 \min 1 \% \mathrm{~B}, 5 \% \mathrm{~B}$ at $9 \min , 7 \% \mathrm{~B}$ at $11 \mathrm{~min}, 10 \% \mathrm{~B}$ at $13 \mathrm{~min}, 15 \% \mathrm{~B}$ at $32 \mathrm{~min}, 40 \% \mathrm{~B}$ at $38 \mathrm{~min}, 50 \% \mathrm{~B}$ at $43 \mathrm{~min}, 100 \% \mathrm{~B}$ at 50 $\min , 100 \% \mathrm{~B}$ at $60 \mathrm{~min}, 1 \% \mathrm{~B}$ at $61 \mathrm{~min}$, and a 12 -min re-equilibration at $1 \% \mathrm{~B}$ before the next injection. The column effluent was diverted to waste for the first 2 min of analysis.

Gas phase ions were generated using an electrospray ionization source operated in positive mode. The capillary voltage was $4500 \mathrm{~V}$, drying temperature $275^{\circ} \mathrm{C}$, and $\mathrm{N}_{2}$ drying and nebulizing gases were $5 \mathrm{~L} \mathrm{~min}^{-1}$ and $1 \mathrm{bar}$, respectively. Mass spectra were collected over a range of 110-600 $\mathrm{m} / \mathrm{z}$. Ions were selected for collision induced dissociation over a $3 \mathrm{~s}$ cycle time using a preferred mass list constructed using the MODOMICS database. ${ }^{1}$ 

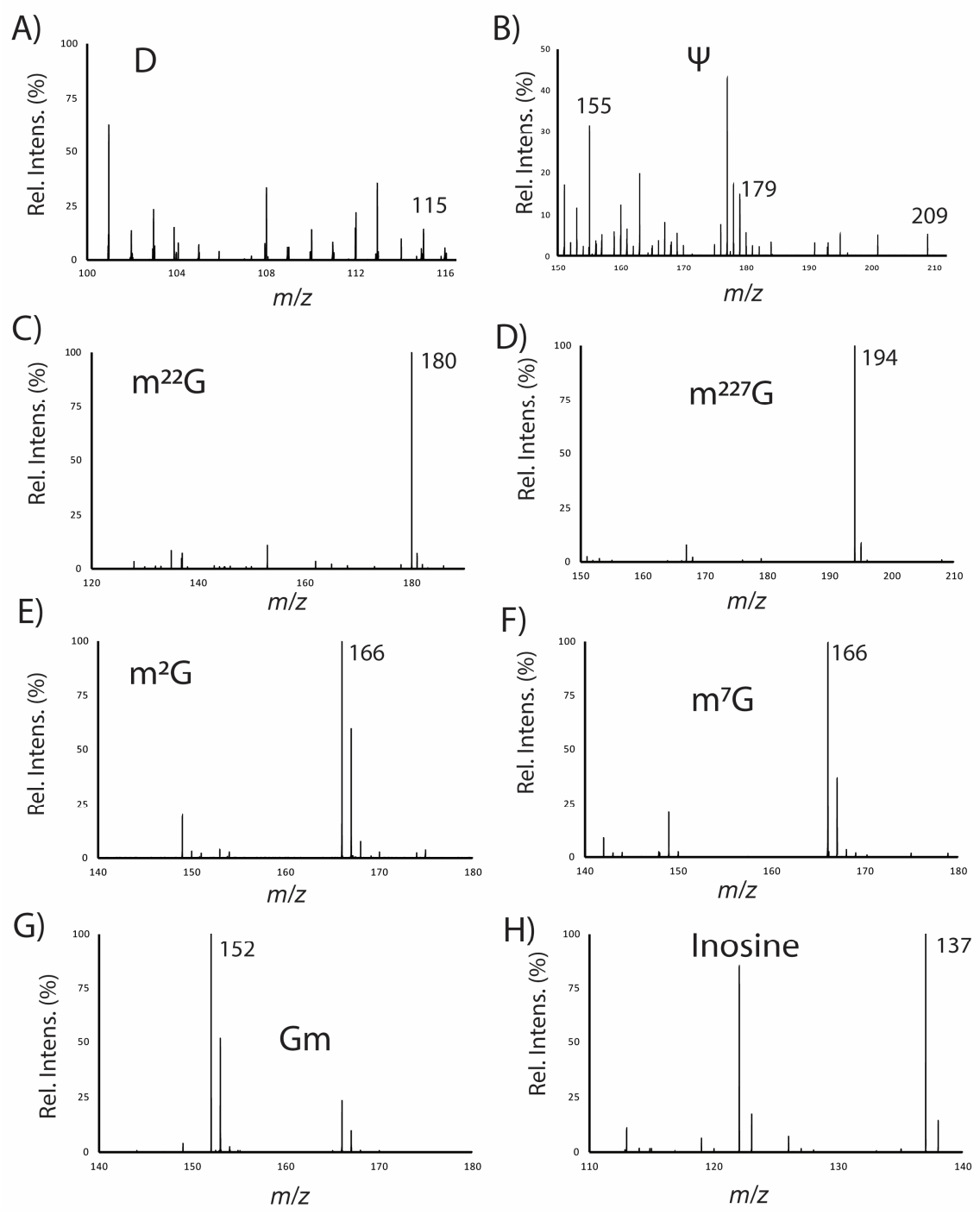

Figure S1 (continued on next page). 

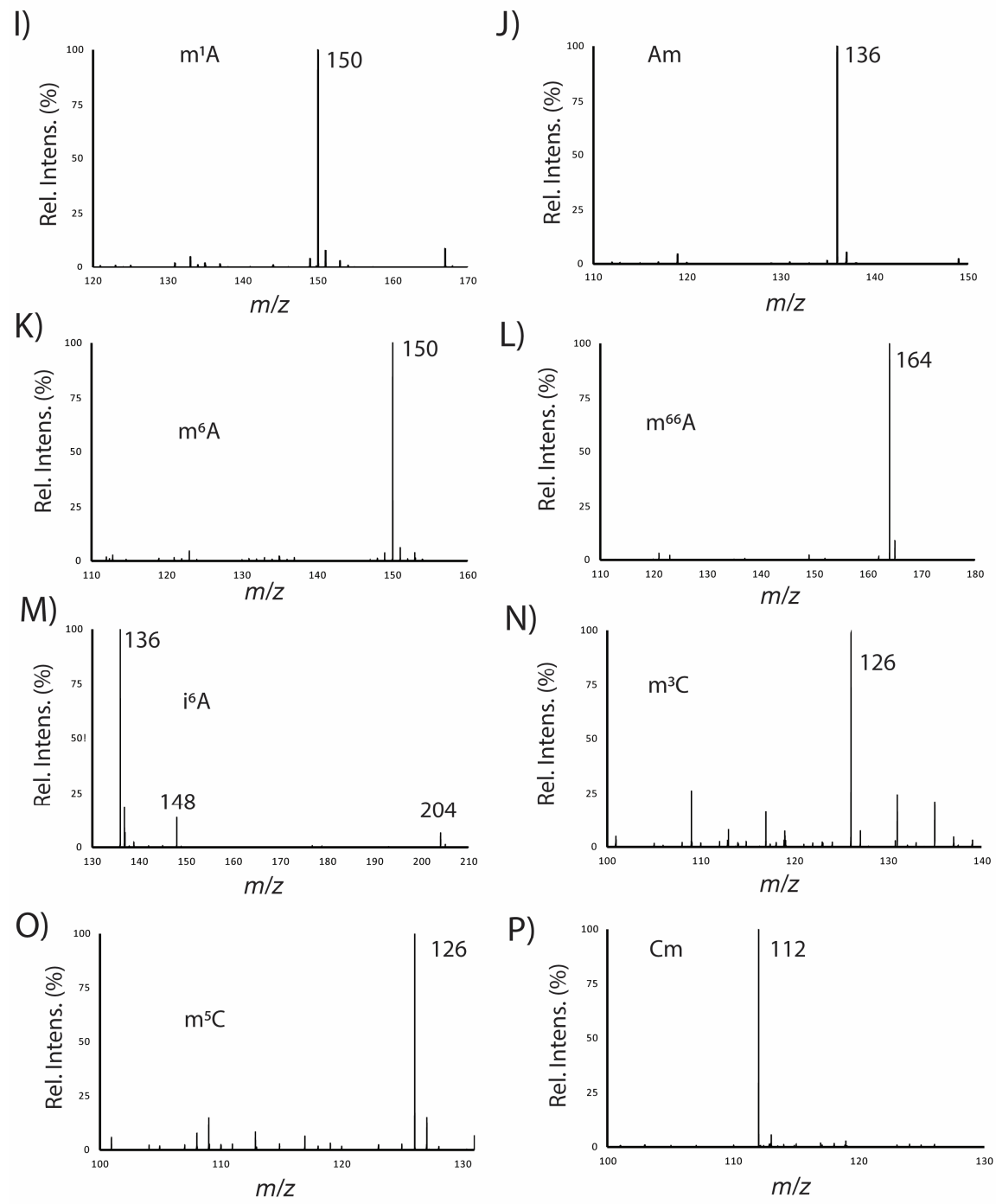

Figure S1. Tandem mass spectra for modified nucleosides in single neurons. Panels A-P were obtained by performing collision induced dissociation on the following parent ions: $\mathrm{m} / \mathrm{z} 247.0910$ (dihydrouridine, D), 245.0751 (pseudouridine, $\Psi), 312.1288\left(\mathrm{~N} 2, \mathrm{~N} 2\right.$-dimethylguanosine, $\left.\mathrm{m}^{22} \mathrm{G}\right)$, 326.1442 (N2,N2,N7-trimethylguanosine, $\left.\mathrm{m}^{227} \mathrm{G}\right), 298.1165$ (N2-methylguanosine, $\left.\mathrm{m}^{2} \mathrm{G}\right)$, 298.1134 (N7-methylguanosine, $\mathrm{m}^{7} \mathrm{G}$ ), 298.1173 (2'-O-methylguanosine, Gm), 269.0855 (inosine, I), 282.1200 (N1-methyladenosine, $\mathrm{m}^{1} \mathrm{~A}$ ), 282.1229 (2'-O-methyladenosine, Am), 282.1229 (N6-methyladenosine, m6 A), 296.1379 (N6,N6-dimethyladenosine, $\mathrm{m}^{66} \mathrm{~A}$ ), 336.1648 (N6-isopentenyladenosine, $\left.\quad \mathrm{i}^{6} \mathrm{~A}\right), \quad 258.1067 \quad\left(\mathrm{~N} 3\right.$-methylcytidine, $\left.\quad \mathrm{m}^{3} \mathrm{C}\right), \quad 258.1122 \quad$ (5methylcytidine, $\mathrm{m}^{5} \mathrm{C}$ ), and 258.1087 (2'-O-methylcytidine, $\mathrm{Cm}$ ). 


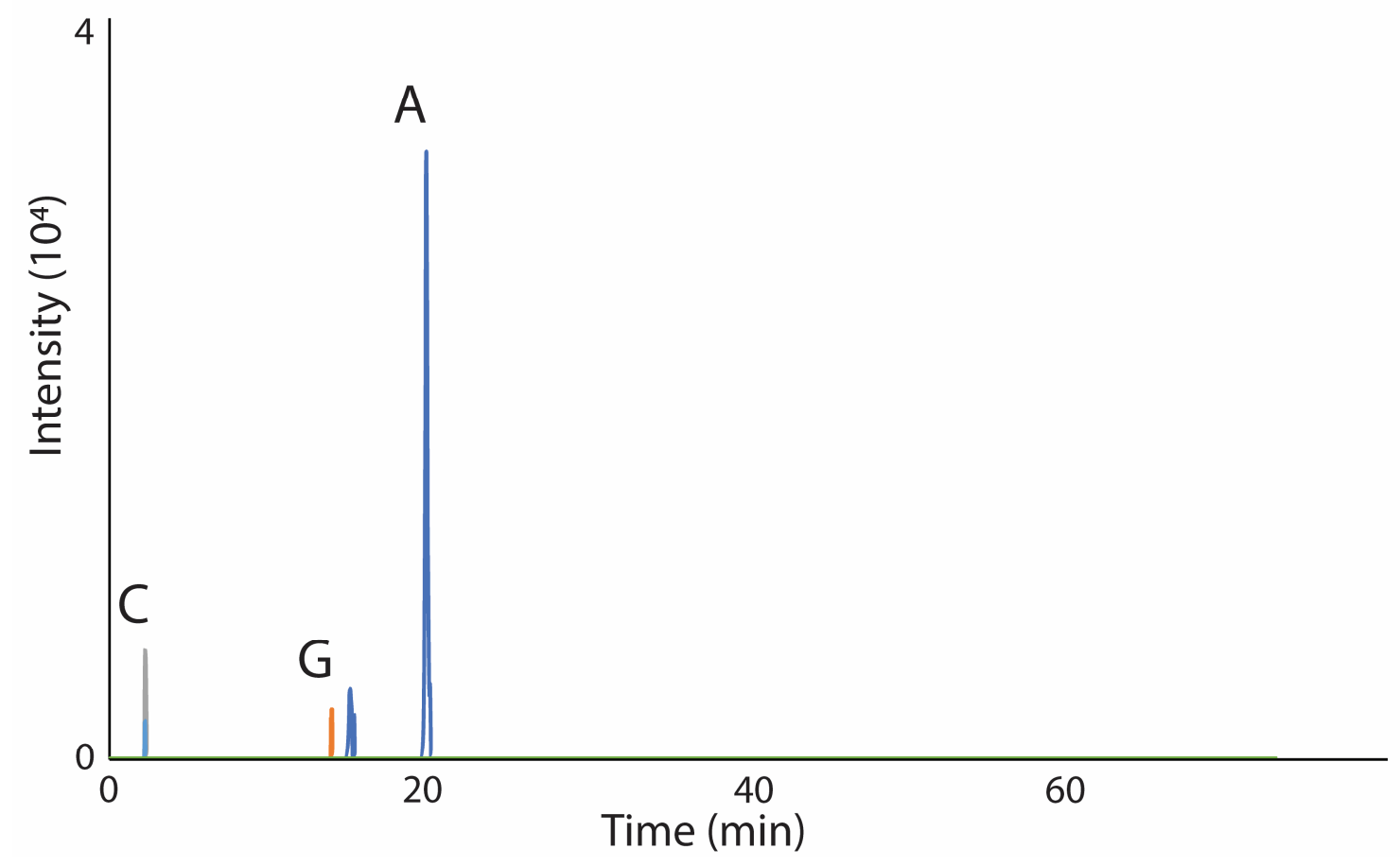

Figure S2. A single LP11 neuron was lysed and incubated in ammonium acetate buffer without digestive enzymes for $3 \mathrm{~h}$ at $37^{\circ} \mathrm{C}$. Extracted ion chromatograms (EICs) for canonical nucleosides cytidine ( $\mathrm{m} / \mathrm{z} 244.09 \pm 0.01$, gray trace), uridine $(\mathrm{m} / \mathrm{z} 245.0773 \pm 0.01$, light blue trace), guanosine $(\mathrm{m} / \mathrm{z} 284.10 \pm 0.01$, orange trace), adenosine $(\mathrm{m} / z 268.11 \pm 0.01$, dark blue trace), and position isomers of methyladenosine $(\mathrm{m} / z 282.12 \pm 0.01$, green trace). Uridine and methyl adenosine isomers were not detected. $\mathrm{C}$ - cytidine, $\mathrm{G}$ - guanosine, $\mathrm{A}$ - adenosine.

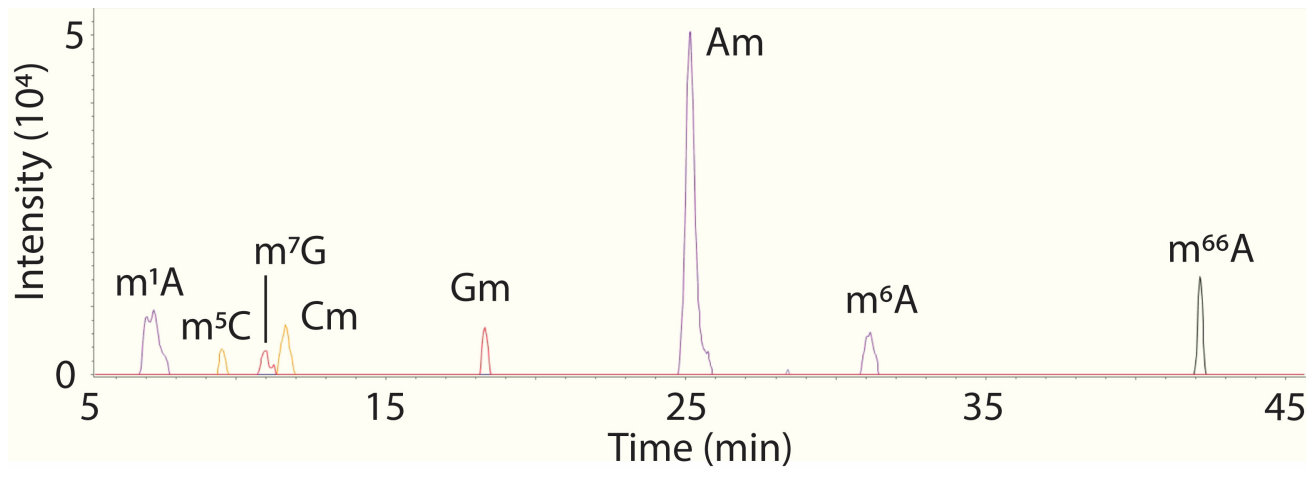

Figure S3. Overlaid EICs for methylated and $\mathrm{CD}_{3}$-labeled nucleosides following spiking a single cell sample with $3 \mathrm{mM} \mathrm{CD}$-Met immediately prior to SNRMA-MS. No $\mathrm{CD}_{3}$-labeled nucleosides were detected; thus, they are not indicated in the figure. EICs for $\mathrm{CD}_{3}$-labeled nucleosides are as follows: $m / z 261.13 \pm 0.01\left(\mathrm{CD}_{3}-\mathrm{m}^{5} \mathrm{C}\right.$ and $\left.\mathrm{CD}_{3}-\mathrm{Cm}\right), m / z 285.14 \pm 0.01\left(\mathrm{CD}_{3}-\mathrm{m}^{1} \mathrm{~A}_{2} \mathrm{CD}_{3}-\mathrm{Am}\right.$, and $\left.\mathrm{CD}_{3}-\mathrm{m}^{6} \mathrm{~A}\right), m / z 301.13 \pm 0.01\left(\mathrm{CD}_{3}-\mathrm{m}^{7} \mathrm{G}\right.$ and $\left.\mathrm{CD}_{3}-\mathrm{Gm}\right), m / z 302.17 \pm 0.01\left(\left(\mathrm{CD}_{3}\right)_{2}-\mathrm{m}^{66} \mathrm{~A}\right)$. Due to low noise and small signals, no baselines are visible. 

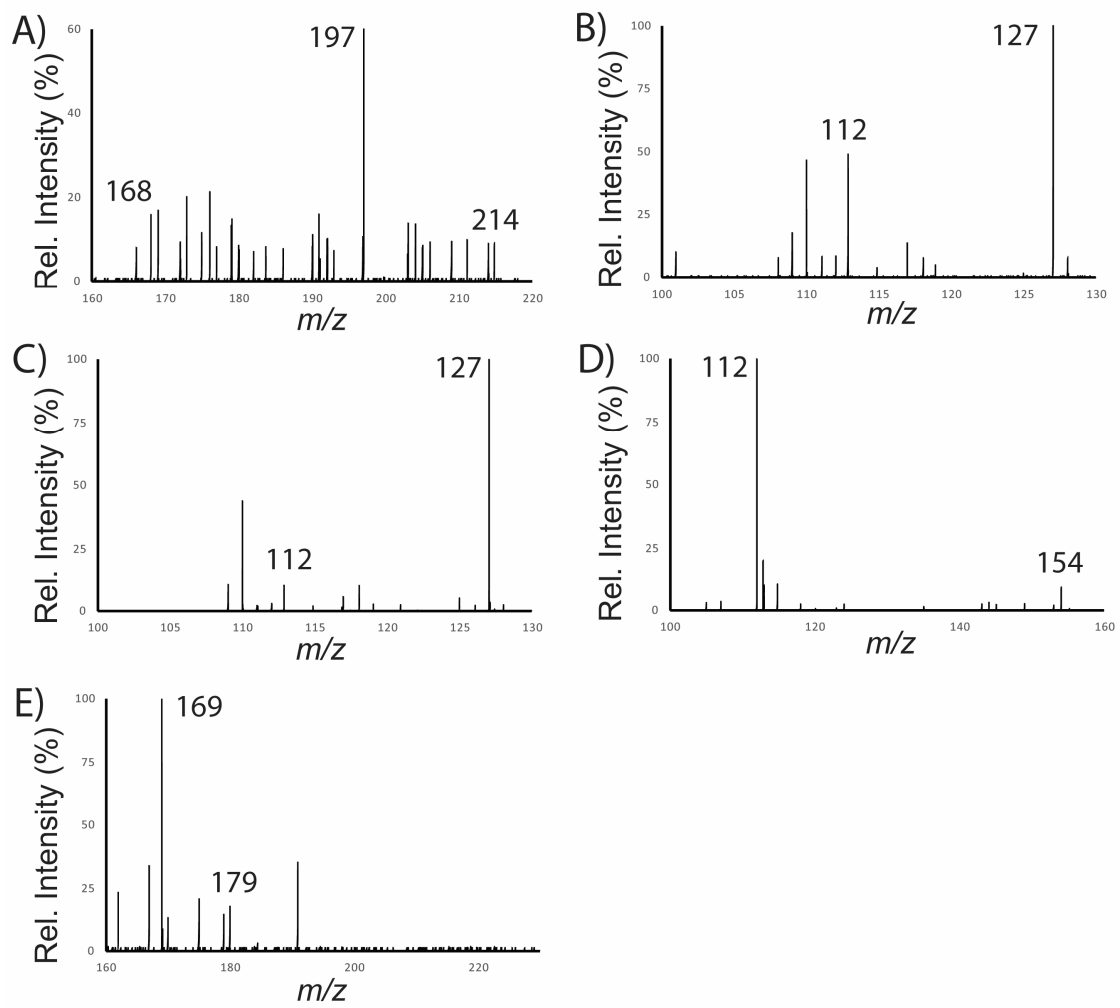

Figure S4. Tandem mass spectra for additional modified nucleosides detected in bulk abdominal ganglia samples. Panels A-E were obtained by CID of parent ions at $\mathrm{m} / \mathrm{z}$ 346.1251 (3-(3-amino3-carboxypropyl)uridine, acp3U), 259.0930 (5-methyluridine, m5U), 259.0878 (3-methyluridine, $\mathrm{m} 3 \mathrm{U}$ ), 286.1041 (N4-acetylcytidine, ac4C), and 259.0892 (methylpseudouridine, $\mathrm{m}^{1} \Psi$ or $^{3} \Psi$ ), respectively. 

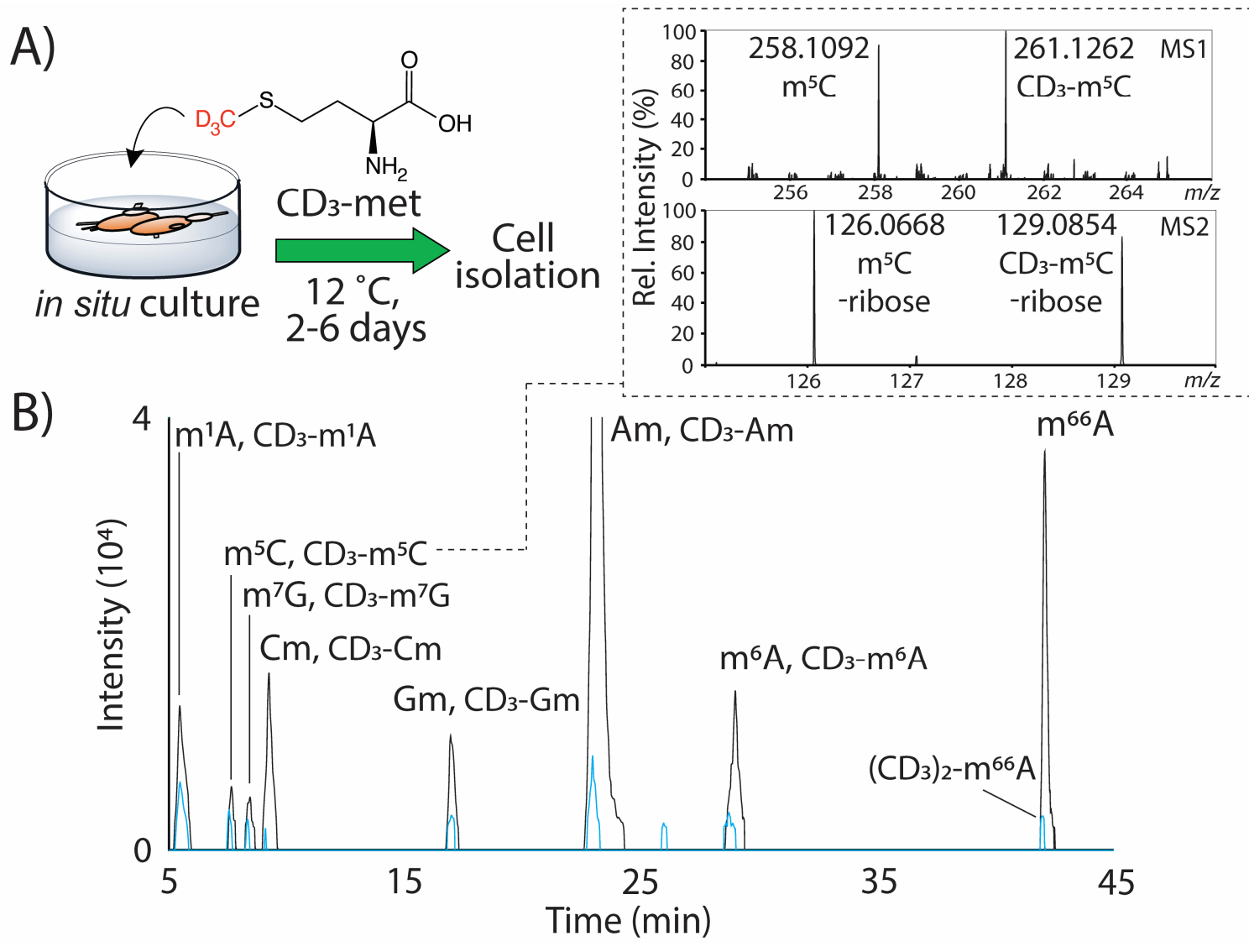

Figure S5. Stable isotope labeling of RNA in single neurons. (A) Workflow involving in situ culture with $\mathrm{CD}_{3}$-Met. (B) Overlaid EICs for $\mathrm{CD}_{3}$-methylated nucleosides (blue traces) and unlabeled counterparts (black traces). Analyzed LP11 neuron was in situ incubated with ASW and $\mathrm{CD}_{3}$-Met for $6 \mathrm{~d}$. EICs for $\mathrm{CD}_{3}$-labeled nucleosides are as follows: $m / z 261.13 \pm 0.01\left(\mathrm{CD}_{3}-\mathrm{m}^{5} \mathrm{C}\right.$ and $\left.\mathrm{CD}_{3}-\mathrm{Cm}\right), m / z 285.14 \pm 0.01\left(\mathrm{CD}_{3}-\mathrm{m}^{1} \mathrm{~A}, \mathrm{CD}_{3}-\mathrm{Am}\right.$, and $\left.\mathrm{CD}_{3}-\mathrm{m}^{6} \mathrm{~A}\right), m / z 301.13 \pm 0.01\left(\mathrm{CD}_{3}-\right.$ $\mathrm{m}^{7} \mathrm{G}$ and $\left.\mathrm{CD}_{3}-\mathrm{Gm}\right), \mathrm{m} / z$ 302.17 $\pm 0.01\left(\left(\mathrm{CD}_{3}\right)_{2}-\mathrm{m}^{66} \mathrm{~A}\right)$. The inset shows MS1 and MS2 spectra for $\mathrm{m}^{5} \mathrm{C}$ and stable isotope-labeled analog, $\mathrm{CD}_{3}-\mathrm{m}^{5} \mathrm{C}$. Isolation window permitted fragmentation of both labeled and unlabeled parent ions. 


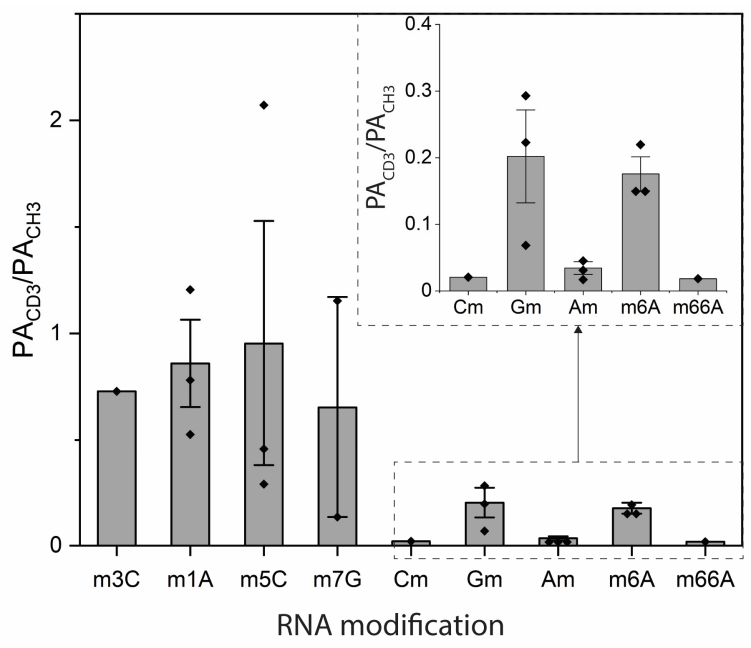

Figure S6. Peak area ratios of $\mathrm{CD}_{3}$-labeled and unlabeled methylated nucleosides for 6-d in situ culture of LP11. Data points represent individual neurons. Mean \pm 1 standard deviation is shown when possible $(\mathrm{n}=3)$.

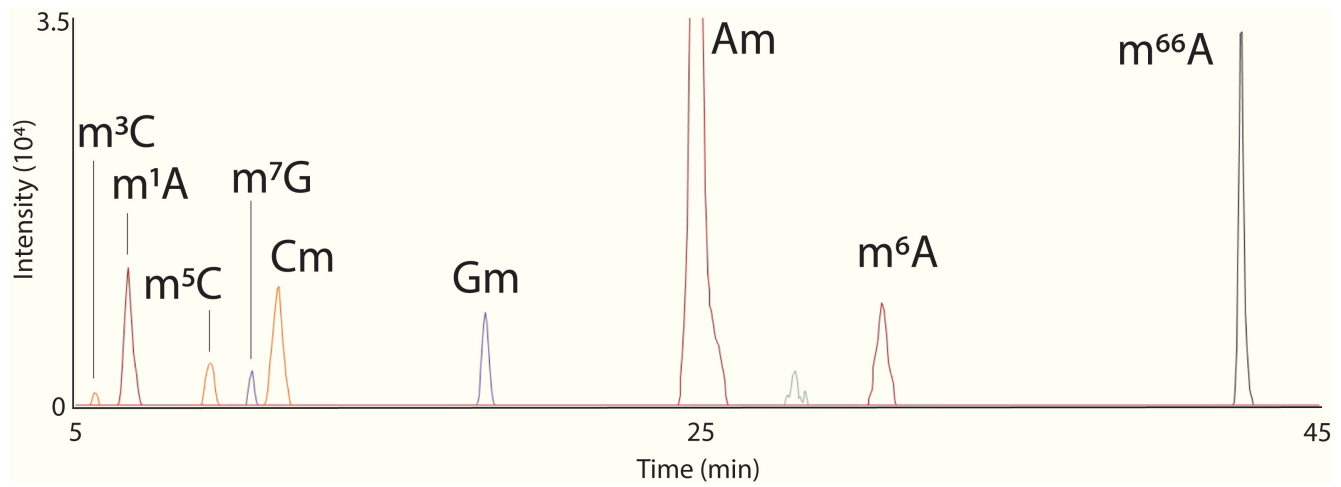

Figure S7. Overlaid EICs for unlabeled and $\mathrm{CD}_{3}$-methylated nucleosides following in situ culture of LP11 with $\mathrm{CD}_{3}-\mathrm{Met}$ and actinomycin $\mathrm{D}$ in situ for $6 \mathrm{~d}$ at $12{ }^{\circ} \mathrm{C}$. No peaks were detected for $\mathrm{CD}_{3}$ methylated nucleosides; thus, they are not indicated in the figure. EICs for $\mathrm{CD}_{3}$-labeled nucleosides are as follows: $m / z 261.13 \pm 0.01\left(\mathrm{CD}_{3}-\mathrm{m}^{5} \mathrm{C}\right.$ and $\left.\mathrm{CD}_{3}-\mathrm{Cm}\right), m / z 285.14 \pm 0.01\left(\mathrm{CD}_{3}-\right.$ $\mathrm{m}^{1} \mathrm{~A}, \mathrm{CD}_{3}-\mathrm{Am}$, and $\left.\mathrm{CD}_{3}-\mathrm{m}^{6} \mathrm{~A}\right), m / z 301.13 \pm 0.01\left(\mathrm{CD}_{3}-\mathrm{m}^{7} \mathrm{G}\right.$ and $\left.\mathrm{CD}_{3}-\mathrm{Gm}\right), m / z 302.17 \pm 0.01$ $\left(\left(\mathrm{CD}_{3}\right)_{2}-\mathrm{m}^{66} \mathrm{~A}\right)$. 


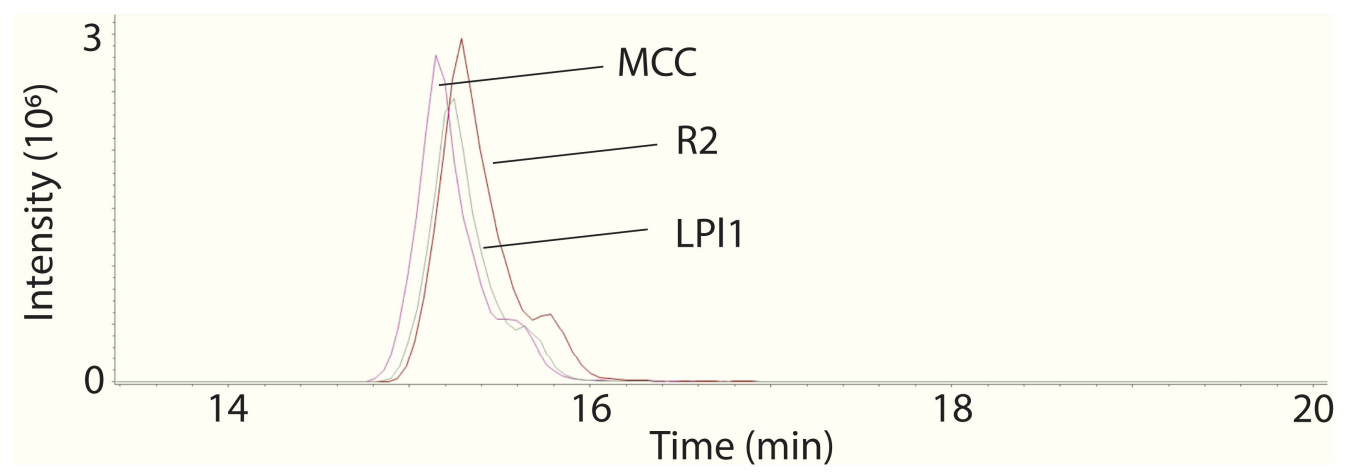

Figure S8. Representative EICs for $\mathrm{CD}_{3}$-Met $(\mathrm{m} / z$ 153.08 \pm 0.01$)$ accumulation in different identified neurons after $96 \mathrm{~h}$.
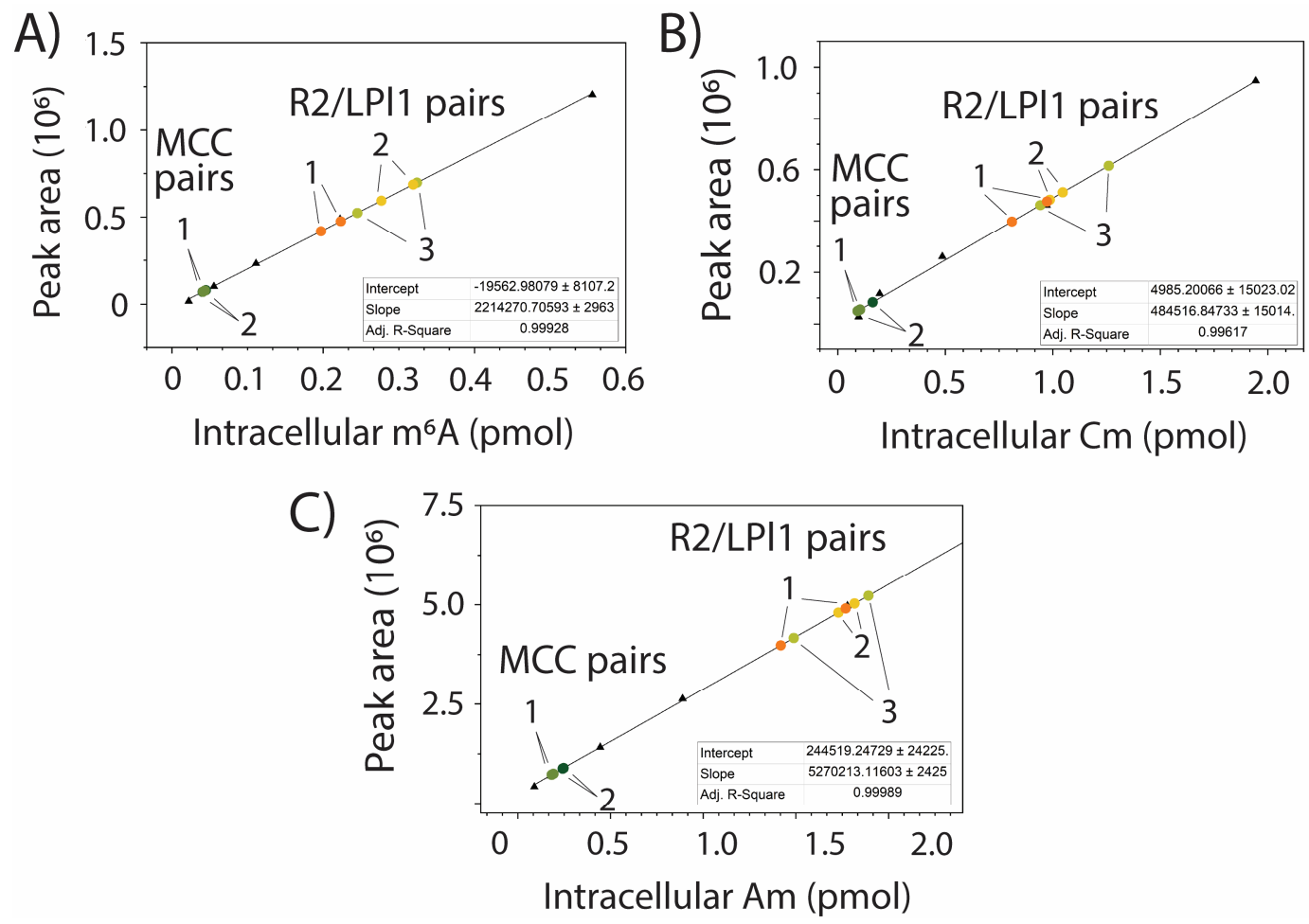

Figure S9. Calibration curves for $\mathrm{m}^{6} \mathrm{~A}, \mathrm{Cm}$, and Am with results of MCC and R2/LP11 neuron quantitative measurements using SNRMA-MS added to the curves. Cell pairs indicate that those neurons were derived from the same animal. Calibration points are represented with triangles, while the circles indicate single neurons. 
Table S1. Abbreviations used in Figure 1D and text as well as LC retention characteristics and $\mathrm{m} / \mathrm{z}$ values used for generating EICs for modified nucleosides.

\begin{tabular}{llrr}
\hline & Abbreviation & $\begin{array}{l}\text { Elution } \\
\text { order (C18) }\end{array}$ & \multicolumn{2}{l}{$\begin{array}{l}\mathrm{m} / z \text { for } \\
\mathrm{EIC}^{a}\end{array}$} \\
\hline RNA modification & $\mathrm{D}$ & 1 & 247.09 \\
pseudouridine & $\Psi$ & 2 & 245.08 \\
N3-methylcytidine & $\mathrm{m}^{3} \mathrm{C}$ & 3 & 258.11 \\
N1-methyladenosine & $\mathrm{m}^{1} \mathrm{~A}$ & 4 & 282.12 \\
N7-methylguanosine & $\mathrm{m}^{7} \mathrm{G}$ & 5 & 298.12 \\
2'-O-methylcytidine & $\mathrm{Cm}$ & 6 & 258.11 \\
2'-O-methylguanosine & $\mathrm{Gm}$ & 7 & 298.12 \\
N2-methylguanosine & $\mathrm{m}^{2} \mathrm{G}$ & 8 & 298.12 \\
N2,N2,N7-trimethylguanosine & $\mathrm{m}^{227} \mathrm{G}$ & 9 & 326.15 \\
N2,N2,-dimethylguanosine & $\mathrm{m}^{22} \mathrm{G}$ & 10 & 312.13 \\
2'-O-methyladenosine & $\mathrm{Am}^{6}$ & 11 & 282.12 \\
N6-methyladenosine & $\mathrm{m}^{6} \mathrm{~A}$ & 12 & 282.12 \\
N6,N6-dimethyladenosine & $\mathrm{m}^{66} \mathrm{~A}$ & 13 & 296.14 \\
N6-isopentenyladenosine & $\mathrm{i}^{6} \mathrm{~A}$ & 14 & 336.17 \\
\hline
\end{tabular}

${ }^{a}$ EICs were plotted in Compass DataAnalysis 4.4 software with a $\pm 0.01 \mathrm{~m} / \mathrm{z}$ window.

Table S2. Results of blanks and control LP11 neuron measurements. Peak areas for canonical nucleoside signals obtained from ASW blanks and no-enzyme SNRMA-MS measurement of a lysed LP11 neuron. Modified nucleosides were not detected in all samples (see Figure S2).

\begin{tabular}{|c|c|c|c|c|c|c|}
\hline & \multicolumn{5}{|c|}{ raw peak area } & \multirow[b]{2}{*}{$\begin{array}{r}\text { Average \% of } \\
\text { SNRMA-MS } \\
\text { signal from blank } \\
\end{array}$} \\
\hline Nucleoside & $\begin{array}{r}\text { ASW } \\
\text { blank } 1\end{array}$ & $\begin{array}{r}\text { ASW } \\
\text { blank } 2 \\
\end{array}$ & $\begin{array}{r}\text { ASW } \\
\text { blank } 3\end{array}$ & $\begin{array}{r}\text { No } \\
\text { enzyme } \\
\text { blank } \\
\end{array}$ & $\begin{array}{r}\text { SNMRA-MS } \\
\text { signal for } \\
\text { LP11 } \\
\end{array}$ & \\
\hline $\mathrm{C}$ & 41293 & nd & 20787 & 48335 & 2106062 & 1.75 \\
\hline $\mathrm{U}$ & nd & nd & nd & nd & 1648062 & 0.00 \\
\hline G & 12387 & nd & 4671 & 17563 & 7072973 & 0.16 \\
\hline A & 351180 & 161030 & 261735 & 410299 & 39050239 & 0.76 \\
\hline
\end{tabular}


Table S3. Peak areas for RNA modifications detected in R2 neurons and the remaining abdominal ganglion, normalized to canonical $\mathrm{C}$, $\mathrm{U}$, and $\mathrm{G}$ nucleosides. R2 neuron was isolated from the abdominal ganglion and both samples analyzed.

\begin{tabular}{|c|c|c|c|c|c|c|c|c|c|c|c|c|c|c|}
\hline $\begin{array}{l}\text { RNA } \\
\text { modification }\end{array}$ & R2-1 & R2-2 & R2-3 & R2-4 & R2-5 & R2-6 & R2-7 & $\mathrm{Ab} g \mathrm{~g}$ & $\mathrm{Ab} g \mathrm{~g}$ & $\mathrm{Ab} g \mathrm{~B}$ & $\mathrm{Abg} 4$ & $\mathrm{Ab} g 5$ & $\mathrm{Ab} g 6$ & $\mathrm{Ab} \mathrm{g} 7$ \\
\hline$\Psi$ & 2.771 & 2.327 & 2.515 & 1.571 & 2.426 & 2.511 & 2.267 & 3.636 & 3.478 & 3.898 & 3.833 & 3.649 & 3.461 & 3.532 \\
\hline $\mathrm{m}^{3} \mathrm{C}$ & 0.277 & 0.477 & 0.263 & 0.061 & 0.318 & $\mathrm{nd}^{a}$ & nd & 0.744 & 0.452 & 0.810 & 0.184 & 0.027 & 0.289 & 0.335 \\
\hline $\mathrm{m}^{1} \mathrm{~A}$ & 5.011 & 5.811 & 4.835 & 3.690 & 6.107 & 4.729 & 4.282 & 5.172 & 3.871 & 5.726 & 4.893 & 5.002 & 3.871 & 3.889 \\
\hline $\mathrm{m}^{7} \mathrm{G}$ & 0.884 & 1.037 & 0.929 & 0.311 & 0.913 & 0.769 & 0.721 & 1.030 & 0.728 & 1.026 & 0.578 & 0.552 & 0.621 & 0.609 \\
\hline $\mathrm{Cm}$ & 2.076 & 1.633 & 1.832 & 1.395 & 1.407 & 1.712 & 1.656 & 1.952 & 1.612 & 1.562 & 1.933 & 1.664 & 1.856 & 1.765 \\
\hline $\mathrm{Gm}$ & 1.418 & 1.096 & 1.274 & 1.079 & 1.734 & 1.588 & 1.230 & 1.911 & 1.690 & 1.934 & 1.912 & 1.852 & 1.716 & 1.630 \\
\hline $\mathrm{m}^{2} \mathrm{G}$ & 0.282 & 0.253 & 0.298 & 0.036 & 0.265 & 0.140 & nd & 0.380 & 0.293 & 0.444 & 0.297 & 0.398 & 0.319 & 0.274 \\
\hline $\mathrm{m}^{227} \mathrm{G}$ & 0.302 & 0.329 & 0.505 & 0.108 & 0.319 & nd & nd & 0.318 & 0.310 & 0.286 & 0.256 & 0.342 & 0.195 & 0.139 \\
\hline $\mathrm{m}^{22} \mathrm{G}$ & 0.186 & 0.071 & 0.053 & nd & 0.190 & nd & nd & 0.334 & 0.295 & 0.394 & 0.306 & 0.345 & 0.246 & 0.230 \\
\hline Am & 13.306 & 11.112 & 14.368 & 13.839 & 16.061 & 19.286 & 17.477 & 11.362 & 9.961 & 10.178 & 14.542 & 12.982 & 11.448 & 11.989 \\
\hline $\mathrm{m}^{6} \mathrm{~A}$ & 2.165 & 1.673 & 2.118 & 1.933 & 2.528 & 2.938 & 2.278 & 2.159 & 1.766 & 1.922 & 2.854 & 2.697 & 2.205 & 2.434 \\
\hline $\mathrm{m}^{66} \mathrm{~A}$ & 2.014 & 1.449 & 2.344 & 1.778 & 1.789 & 2.586 & 2.547 & 2.225 & 1.788 & 1.960 & 2.295 & 1.861 & 1.687 & 1.821 \\
\hline$i^{6} \mathrm{~A}$ & 0.299 & 0.348 & 0.328 & 0.043 & 0.172 & 0.050 & 0.073 & 0.267 & 0.186 & 0.267 & 0.130 & 0.187 & 0.132 & 0.119 \\
\hline
\end{tabular}

${ }^{a}$ not detected.

Table S4. Diameters and calculated intracellular volumes of studied R2, LP11, and left and right MCCs (LMCC and RMCC).

\begin{tabular}{ccccccc}
\hline Animal & Cell & $\begin{array}{c}\text { diameter 1 } \\
(\mu \mathrm{m})\end{array}$ & $\begin{array}{c}\text { diameter 2 } \\
(\mu \mathrm{m})\end{array}$ & $\begin{array}{c}\text { diameter 3 } \\
(\mu \mathrm{m})\end{array}$ & $\begin{array}{c}\text { est. spherical } \\
\text { volume }(\mu \mathrm{L})\end{array}$ & $\begin{array}{c}\text { est. ellipsoid } \\
\text { volume }(\mu \mathrm{L})\end{array}$ \\
\hline 1 & LP11 & 463.75 & 509.27 & 373.75 & 0.0069 & 0.0462 \\
1 & R2 & 503.81 & 603.21 & 408.59 & 0.0115 & 0.0650 \\
2 & LP11 & 559.02 & 439.37 & 480.69 & 0.0091 & 0.0618 \\
2 & R2 & 404.46 & 449.79 & 431.2 & 0.0048 & 0.0411 \\
3 & LP11 & 487.37 & 413.22 & 443.42 & 0.0061 & 0.0468 \\
3 & R2 & 555.69 & 363.46 & 508.15 & 0.0090 & 0.0537 \\
1 & LMCC & 291.77 & 281.26 & 240.37 & 0.0130 & 0.0103 \\
1 & RMCC & 289.02 & 275.7 & 244.6 & 0.0013 & 0.0102 \\
2 & LMCC & 244.81 & 240.31 & 235.55 & 0.0008 & 0.0073 \\
2 & RMCC & 244.6 & 240.16 & 244.48 & 0.0008 & 0.0075 \\
\hline
\end{tabular}




\section{REFERENCE}

(1) Boccaletto, P.; Machnicka, M. A.; Purta, E.; Piątkowski, P.; Bagiński, B.; Wirecki, T. K.; de Crécy-Lagard, V.; Ross, R.; Limbach, P. A.; Kotter, A.; Helm, M.; Bujnicki, J. M. MODOMICS: A Database of RNA Modification Pathways. 2017 Update. Nucleic Acids Res. 2017, 46 (D1), D303-D307. https://doi.org/10.1093/nar/gkx1030. 